\title{
Toxic Shock Syndrome Toxin-1
}

National Cancer Institute

\section{Source}

National Cancer Institute. Toxic Shock Syndrome Toxin-1. NCI Thesaurus. Code C1086.

Staphylococcal toxic shock syndrome toxin 1. A superantigen with the potential to suppress plasma cell differentiation and antibody responsiveness. Potent pyrogen, induces T lymphocyte proliferation, requiring interleukin 1 release from macrophages, suppresses immunoglobulin production, enhances endotoxin shock, and enhances hypersensitivity skin reactions. Causes staphylococcal toxic shock syndrome (TSS). 